\title{
Pratiques
}

Linguistique, littérature, didactique

141-142 | 2009

La synonymie

\section{Jalousie et envie : l'affectivité tout en nuances}

\section{Arkadiusz Koselak}

\section{(2) OpenEdition}

Journals

Édition électronique

URL : https://journals.openedition.org/pratiques/1337

DOI : $10.4000 /$ pratiques. 1337

ISSN : 2425-2042

\section{Éditeur}

Centre de recherche sur les médiations (CREM)

\section{Édition imprimée}

Date de publication : 15 juin 2009

Pagination : 165-178

\section{Référence électronique}

Arkadiusz Koselak, « Jalousie et envie : I'affectivité tout en nuances », Pratiques [En ligne], 141-142 | 2009, mis en ligne le 20 juin 2014, consulté le 10 mars 2023. URL : http://journals.openedition.org/ pratiques/1337 ; DOI : https://doi.org/10.4000/pratiques.1337 


\title{
Jalousie et envie : l'affectivité tout en nuances
}

\author{
Arkadiusz Koselak
}

Jalousie et envie sont deux noms dont certaines acceptions décrivent la même situation : l'expérienceur perçoit la présence, chez autrui, d'un bien ou d'une qualité qu'il n'a pas lui-même et qu'il aimerait avoir. Les locuteurs naïfs interrogés informellement sont très ambivalents au sujet des rapports de ces deux concepts, mais s'avouent souvent incapables d'en expliciter les différences. Les dictionnaires, comme par exemple Le Petit Robert, présentent la jalousie et l'envie comme des synonymes.

Décider d'une éventuelle synonymie de la jalousie et de l'envie nécessite une interrogation sur le concept de la synonymie-même. Cette notion, bien qu'elle semble très évidente, n'est pas vraiment facile à manipuler. La synonymie est-elle une relation lexicale ou une relation de sens ? Cette question ne joue pas sur les mots, y répondre oblige à préciser la notion-même de sens. Sans prétention de résoudre ici le problème de la synonymie, je vais rappeler quelques questions méthodologiques importantes concernant cette notion et poser des repères nécessaires pour traiter la jalousie et l'envie.

Mais jalousie et envie sont polysémiques et leurs rapports, comme c'est souvent le cas, ne portent pas sur toutes les valeurs. Avant de pouvoir s'interroger sur les relations entre les deux concepts, il faudra les envisager séparément mais selon les mêmes principes afin d'obtenir des données comparables. Une fois ces données obtenues et comparées pour mettre en évidence les similitudes et les ressemblances entre la jalousie et l'envie, je procéderai à la vérification de substitution de deux concepts dans les énoncés réellement produits et je tenterai de préciser la relation qui unit ces deux concepts.

Dans la mesure où l'on peut considérer que la synonymie totale n'existe pas, il faut s'interroger sur l'opérationnalité de la notion de synonymie partielle. Comment décider quel degré de cette différence est acceptable pour que l'on puisse parler encore d'une synonymie ? Cruse (2002) fait observer que malgré le caractère scalaire de la synonymie, il y a un consensus intuitif pour dire si telle ou telle paire des mots est synonyme. Pour le besoin d'analyse du couple envie/jalousie, je retiendrai la condition suivante : pour que deux concepts puissent être envisagés 
comme synonymes, il faut d'une part qu'ils appartiennent au même domaine conceptuel, donc que l'on puisse leur trouver un même hypéronyme, et d'autre part, qu'une partie fondamentale de leur contenu sémantique soit commune. J'entends par «partie fondamentale » la composante déterminante et inaliénable du sens. Bien que peu précise, cette formulation peut orienter les analyses. Elle sera réévaluée infra sur le matériel linguistique concret.

Du point de vue formel, le test linguistique le plus important de la synonymie est la substitution. On substitue un mot à un autre dans un énoncé et l'énoncé obtenu doit avoir soit le même sens soit les mêmes conditions de vérité. Je retiendrai ici le critère d'identité de sens avec la même réserve que celle adressée aux synonymes, le sens de deux énoncés ainsi obtenus ne peut être identique, il ne peut être éventuellement que proche. Niklas-Salminen (1997), précise que, pour que la substitution soit valable, les synonymes doivent appartenir à la même classe grammaticale. Nous verrons que dans le cas de la jalousie et de l'envie cette précision est importante.

Au lieu de procéder d'emblée à des tests de substitution, je vais analyser et décrire tout d'abord le contenu sémantique de la jalousie et de l'envie. La description effectuée selon un modèle commun permettra ensuite de dire précisément quels sont les changement de sens opérés lors de la substitution.

\section{Analyse sémantique de jalousie et envie}

\subsection{Jalousie}

La jalousie est un affect ${ }^{(1)}$. Il n'existe pas en français de moyens formalisés pour prouver l'appartenance d'un concept au domaine affectif, mais on peut appuyer l'intuition par la possibilité de collocation du nom d'affect avec le générique nominal du domaine affectif sentiment de et avec un des génériques verbaux (éprouver et ressentir) suivi du partitif(Koselak, 2007b). La jalousie est considérée intuitivement par les locuteurs naïfs comme un concept affectif et elle accepte les collocations citées. Comme la plupart des concepts affectifs, la jalousie est soumise aux variations de l'intensité et, comme tous les affects, la jalousie est axiologiquement orientée.

Les affects sont des processus complexes qui activent les domaines du penser, du vouloir, du ressentir et du faire. Un affect est la configuration spécifique de composantes cognitive, affective et physique. La composante cognitive est déterminante pour un affect : 1'expérienceur perçoit une situation et effectue un jugement (appraisal) sur cette situation. Conjointement au jugement, l'expérienceur veut quelque chose par rapport à la situation considérée. Ces processus cognitif et volitif s'accompagnent d'un ressenti affectif et, parfois, de comportements et de manifestations internes au corps de l'expérienceur. Les manifestations internes sont difficilement saisissables et dicibles, pour cette raison, elles sont le plus souvent métaphorisées. Je montrerai par la suite comment ces différentes composantes fonctionnent dans le cas de la jalousie.

La jalousie est polysémique et elle a trois valeurs : la convoitise du bien d'autrui (Je suis jaloux de la réussite de Pierre), la crainte de perdre l'exclusivité de la personne aimée (Je suis jaloux de ma femme) et la crainte de perdre un objet qui a

(1) J'utilise dorénavant le terme générique d'affect afin d'éviter les termes émotion et sentiment qui désignent des saisies particulières des affects ( $f$. Koselak, 2007b). 
de la valeur (Il garde jalousement ses secrets). Dans la problématique de la synonymie avec l'envie, seule la première valeur va nous intéresser ${ }^{(2)}$.

Pour décrire la valeur de la jalousie « convoitise du bien d'autrui », je vais partir du postulat énoncé par Harkins \& Wierzbicka (2001) selon lequel les unités linguistiques qui renvoient à des affects sont en fait des « cristallisations » de situations prototypiques dans lesquelles les affects s'actualisent. Ces situations peuvent être formalisées sous la forme de la structure argumentale. Pour la jalousie « convoitise du bien d'autrui » le schéma argumental est : $\mathrm{X}(\mathrm{Y} / \mathrm{Z})$, où $\mathrm{X}$ est expérienceur, $Y$ un objet humain et $Z$, l'objet possédé par $Y$ et convoité par $X$. Un certain nombre de concepts, dont l'envie, se construit autour du même schéma et c'est justement ce schéma qui permet le rapprochement entre la jalousie et l'envie (Koselak, 2008b). Le schéma argumental se manifeste, complètement ou partiellement, dans la structure actancielle des expressions de la jalousie. L'absence ou la présence d'un argument dans la structure actancielle constitue un profilage particulier de l'affect, cette question ne sera pas abordée ici. La valeur de la jalousie qui nous préoccupe peut être exprimée soit par le nom jalousie :

Dans quelle mesure la netteté de son jugement fut-elle obscurcie par la jalousie portée à la gloire d'un rival ? (Frantext)

soit par la structure essive être jaloux :

J'étais très jalouse de tes cheveux, tu sais... (Frantext)

soit enfin, assez rarement, par l'adverbe jalousement :

Je regarde jalousement ceux et celles qui déambulent dans la rue avec ces petites choses arrimées au conduit auditif, pendant que je tente lamentablement de ne pas respirer trop vivement dans l'espoir de faire tenir mes oreillettes toute neuves au moins jusqu'à la fin de la chanson. (Internet)

Il existe également le verbe jalouser, mais il semble rare en français contemporain, du moins métropolitain, il n'en sera pas question ici. La structure argumentale s'exprime de différentes manières dans les structures actancielles de ces unités linguistiques. La structure actancielle dans laquelle la schéma argumental se manifeste de manière la plus complète est l' expression essive être jaloux, il s'agit de l'expression expérientielle de base. Le fait qu'il s'agisse d'une structure essive est important pour la mise en relation avec l'envie.

Nous avons vu que la jalousie concernait la situation dans laquelle un individu $(\mathrm{X})$ convoitait un objet $(\mathrm{Z})$ présent chez un autre individu (Y). La jalousie ne peut pas être envisagée au sujet d'un objet qui n'appartient à personne, les liens entre Y et $\mathrm{Z}$ sont indispensables. Il est possible de produire des énoncés dans lesquels $\mathrm{Y}$ est absent, comme par exemple :

Je suis jaloux de Pierre.

mais l'objet $Z$ doit être inférable ou récupérable dans le co(n)texte. Par contre, les énoncés sans objet $Z$ sont inacceptables :

*Je suis jaloux de la maison.

parce qu'ils présupposeraient que l'on puisse être jaloux d'un objet qui n'appartient à personne.

(2) Pour une description complète de la jalousie, voir Koselak (2008a). 
La composante cognitive de la jalousie peut être décrite de la manière suivante : l'expérienceur perçoit la situation du monde dans laquelle un individu est en possession d'un objet (Z); l'expérienceur n'a pas l'objet en question et il pense que cette situation (Y a Z, X n'a pas Z) est mauvaise. L'expérienceur doit penser également que l'objet $Z$ lui est, du moins théoriquement accessible, par contre il n'est pas nécessaire que l'expérienceur et l'individu Y soient égaux. Il faut préciser que l'expérienceur ne veut pas avoir le même objet que Y, mais un objet du genre de celui que Y possède, il ne s'agit donc pas d'identité mais de similitude. Ensuite, il est propre de chaque « mauvais » affect que l'expérienceur veuille changer la situation qui est à la base de l'affect en question, afin de ne plus le ressentir. Dans la cas de la jalousie, l'expérienceur veut entrer en possession d'un objet du genre de Z (activation du domaine volitionnel), mais sans qu'il en résulte une tendance à l'action. Par ailleurs, l'expérienceur doit considérer que l'objet du genre de Z lui est théoriquement accessible. L'observation des énoncés ne permet pas de déduire une quelconque hostilité de X envers Y qui est mentionnée dans Le Petit Robert. La « cogitation » qui vient d'être décrite s'accompagne d'un ressenti affectif négatif, l'expérienceur « ressent quelque chose de mauvais ». La composante physique est peu spécifique dans le cas de la jalousie, les comportements sont peu nombreux et se concentrent principalement au niveau du visage : regarder, observer jalousement, traits crispés par la jalousie, rire jaloux, coup d'œil jaloux, regard jaloux, mais il existe des manifestations concernant le corps entier : trembler de jalousie, mouvement jaloux, air jaloux. Enfin, il ne faut pas oublier l'ensemble comportamental la scène de jalousie. Les manifestations internes peuvent être regroupées sous la métaphore « la jalousie est parfois comme une maladie», par exemple : la jalousie maladive, une crise de jalousie, la jalousie mortelle et léthargique, la plaie ouverte de la jalousie, paralysé par une jalousie désespérée, intoxiqué par une affection incurable : la jalousie, la démence de la jalousie, l'accès de jalousie, des fureurs de jalousie, dévoré par la jalousie, souffrir de jalousie, la jalousie morbide.

Pour complèter l'invariant conceptuel de lajalousie, il faut ajouter que la jalousie, en plus de son orientation axiologique négative interne (penser et ressentir quelque chose de mauvais), est également considérée comme négative au plan axiologique externe : «les gens pensent que ressentir quelque chose comme cela est mauvais ».

Les observations effectuées peuvent être énoncées sous la forme d'une explicitation verbale qui formalise l'invariant conceptuel (cognitif). Le modèle de l'explicitation, établi par Wierzbicka (1999), est valable pour tout le domaine affectif :

$\mathrm{X}$ a ressenti quelque chose parce que $\mathrm{X}$ a pensé quelque chose

Parfois quelqu'un pense de quelqu'un d'autre :

« cette personne a quelque chose

je n'ai pas de chose de ce genre

je pense que c'est mauvais

je pense que je pourrais avoir quelque chose de ce genre

je voudrais avoir quelque chose de ce genre»"

quand quelqu'un pense cela, il ressent quelque chose de mauvais

$\mathrm{X}$ a ressenti quelque chose comme cela

parce que $\mathrm{X}$ a pensé quelque chose comme cela

L'explicitation verbale est écrite en métalangue sémantique naturelle ${ }^{(3)}$ que je

(3) J'utilise la version française de la métalangue sémantique naturelle de Peeters (2002). Il 
ne présente pas ici ${ }^{(4)}$. Elle se compose d'un cadre commun à tous les affects et d'un contenu présenté comme pensé par un expérienceur (forme de la première personne du présent) qui est propre à chaque affect. Il s'agit d'un genre de scénario cognitif. Je n'ai pas inclus dans l'explicitation la composante physique, elle est peu spécifique pour la jalousie et elle n'est pas indispensable pour la comparaison avec l'envie. Le rapport entre $Y$ et $Z$ est explicité en terme de possession d'un objet. Ce n'est pas toujours le cas, il peut s'agir d'une qualité ou d'une situation, mais la possession au sens matériel semble être prototypique dans la jalousie.

\subsection{Envie}

L'envie est-elle un affect ? sinon qu'est-ce qu'elle est? L'envie c'est quelque chose de bon ou de mauvais? Autant de questions importantes auxquelles il n'est pas facile de trouver de réponses parce que l'envie est un concept relativement peu déterminé sémantiquement.

L'envie est polysémique. Certains dictionnaires, comme par exemple Le Petit Robert, donnent, en premier lieu, une valeur affective : "sentiment de désir mêlé d'irritation et de haine qui anime qqn contre la personne qui possède un bien qu'il $n$ 'a pas » ${ }^{(5)}$, avec l'exemple, fort ancien et gnomique : "Devant la richesse le sentiment le plus ordinaire ce n'est pas le respect, c'est l'envie (Fustel de Coul.) ». Si l'énoncé exemple est censé aider à comprendre la définition, il semble être mal choisi : on peut y percevoir le désir, mais l'irritation et haine ne sont pas visibles. Par ailleurs, l'observation de la base de données contemporaines ${ }^{(6)}$ provenant du corpus Frantext et obtenue sur l'Internet à l'aide du moteur de recherche Google, ne permet pas du tout d'inférer l'existence d'une attitude négative envers la personne qui possède le bien qu'on aimerait avoir.

Dans tous les énoncés avec l'envie et ses dérivés, on repère l'idée du désir, mais ce désir est profilé différemment selon les différentes expressions. Observons tout d'abord les énoncés suivants :

\section{L'envie de pisser me réveilla vers midi. (Frantext)}

J'ai eu le sentiment qu'elle était plus malheureuse que moi, et il m'est venu l'envie de lui caresser le visage. (Frantext)

Je suis très fatigué, j'ai envie de m'étendre par terre. (Frantext)

J'ai envie de vomir. (Frantext)

Ces quatre énoncés peuvent être paraphrasés à l'aide du verbe vouloir, il existe cependant une différence. Tandis que le verbe vouloir présuppose un engagement conscient de l'expérienceur, les expressions de l'envie profilent la volition comme étant indépendante de 1'expérienceur. Ainsi un énoncé comme :

Je veux dormir.

existe une version plus récente (Peeters, 2006), mais les modifications qu' on y a introduites me semblent discutables, notamment en ce qui concerne les primitifs fondamentaux pour le domaine affectif : le verbe ressentir et les adjectifs évaluateurs bon et mauvais (cf. Koselak, à paraître).

(4) Pour la présentation de la métalangue sémantique naturelle voir, par exemple, en français, Peeters (2002), Koselak (2003) ou le site de la métalangue sémantique naturelle : http://www.une.edu.au/lcl/nsm

(5) Cette définition ressemble fort aux descriptions cartésiennes des émotions.

(6) Tous les énoncés utilisés ont été produits après 1945. 
implique que l'expérienceur décide et assume sa volonté d'aller se coucher. Il n'est pas possible d'enchaîner cet énoncé avec un énoncé impliquant le contraire :

* Je veux dormir mais je veux rester éveillé.

Par contre il est tout à fait possible d'opérer cet enchaînement avec les expressions de l'envie :

J'ai envie de dormir / l'envie de dormir m'envahit mais je veux rester éveillé.

Ce qui montre que l'envie, dans cette acception, est indépendante de la conscience de l'expérienceur. Autrement dit, il arrive quelque chose dans l'expérienceur, en dehors et indépendamment de sa conscience. Cette acception de l'envie peut être exprimée soit par le nom envie, soit par la structure avoir envie et elle appartient pleinement au domaine volitionnel.

Il n'est pas possible d'exprimer la valeur que nous venons de voir avec le verbe envier pour des raisons d'influence de la forme sur le sens. Le verbe envier est un verbe transitif à la voie active et ce type de verbes est prototypiquement utilisé pour exprimer des actions. Les affects et d'autres processus mentaux exprimés à l'aide de ce type de verbes présupposent en engagement de l'expérienceur dans le processus exprimé, contrairement aux expressions verbales complexes et les structures essives (Koselak, 2007b). Lorsqu' on substituera la jalousie et l'envie, on aura à faire pratiquement toujours ${ }^{(7)}$ à une différence sémantique liée à l'engagement de l'expérienceur.

Observons maintenant quelques énoncés avec le verbe envier :

J'enviais son succès auprès de filles, plus tard auprès de femmes. (Frantext)

Dans la rue à huit heures du matin : j'envie parfois ces gens qui ont un travail " honnête». (Frantext)

Je vous envie de pouvoir prendre quelques semaines de repos dans cette période qui est pour moi si bouleversée. (Frantext)

On comprendra de ces énoncés que l'expérienceur perçoit une situation du monde dans laquelle quelqu'un est en possession de quelque chose. On peut montrer, à l'aide de l'enchaînement que l'expérienceur souhaiterait avoir le même genre de choses que la personne enviée :

??? J'enviais son succès auprès de filles mais je ne voulais pas avoir un tel succès.

L'impossibilité de cet enchaînement opéré nous montre, à contrario, le désir de l'expérienceur d'avoir une chose semblable. Assez étrangement, le nom envie est relativement peu utilisé pour exprimer cette valeur, en dehors des énoncés gnomiques. Dans tous les emplois que j'ai relevés, je n'ai pas trouvé de trace d'hostilité envers l'autre.

La question qui se pose c'est : est-ce que l'envie est un affect? L'envie accepte d'entrer en collocation avec le générique nominal sentiment de, mais on trouve relativement peu d'occurrences de syntagme obtenu ${ }^{(8)}$. En ce qui concerne les collo-

(7) Sauf des profilages particuliers qui montrent l'envie comme indépendante de l'expérienceur. Cela ne concerne que des emplois nominaux.

(8) 731 résultats obtenus à l'aide de Google. A comparer avec le syntagme sentiment de jalousie : 33.100 résultats et sentiment de haine, pour la comparaison : 60.600 . 
cations avec les génériques verbaux, il y a encore moins de résultats : 21 pour ressentir de l'envie ${ }^{(9)}$ et 31 pouréprouver de l'envie ${ }^{(10)}$. Ajoutons à cela que les locuteurs, interrogés informellement, pensent qu'il n'y a pas de ressenti négatif dans l'envie, contrairement à la jalousie. Comme il ne peut pas y avoir non plus de ressenti positif, l'envie ne peut pas être un affect dans la mesure où tous les affects sont nécessairement valencés en bons et mauvais (MacLean, 1980). On trouve quelques occurrences du verbe envier que l'on peut interpréter en terme de concept affectif mais il s'agit de cas relativement rares. L'envie était peut-être un affect mais elle ne semble plus l'être actuellement ${ }^{(11)}$. Et si l'envie n'est pas un affect, qu'est-ce qu'elle est alors? Il apparaît que l'envie semble appartenir exclusivement aux domaines du vouloir et du penser ${ }^{(12)}$.

Lorsque l'on reprend les deux valeurs que nous venons de voir, on peut dire que seulement la deuxième est en relation sémantique avec la jalousie. Je vais procéder maintenant à l'analyse de cette deuxième valeur. Partons de deux énoncés :

Je vous envie de pouvoir prendre quelques semaines de repos dans cette période qui est pour moi si bouleversée. (Frantext)

Les filles de la blanchisserie enviaient Odette de monopoliser ses regards, ses caresses. (Frantext)

L'envie, dans cette acception, a le même schéma argumental que la valeur correspondante de jalousie: $\mathrm{X}(\mathrm{Y} / \mathrm{Z})$. Un expérienceur perçoit quelque chose (Z) qui est possédé par un autre individu (Y) et souhaiterait avoir la même chose (ou quelque chose dans le même genre). Ainsi par exemple les filles de la blanchisserie (X) voient le succès $(Z)$ d'Odette $(Y)$ et souhaitent avoir du succès également. Cependant, dans le cas de l'envie, cette volonté semble beaucoup moins forte : 1'expérienceur ne veut pas un objet du genre de $\mathrm{Z}$ mais aimerait/souhaiterait en avoir un. Et encore, cette formulation n'est pas suffisamment précise. La schéma argumental de l'envie se matérialise dans la structure actancielle du verbe envier de deux manières : X envie $Y$ et $X$ envie $Z$ à $Y_{D A T}$. La première structure actancielle ressemble à celle de la jalousie, à une différence près, dans l'envie il n'y a pas de médiation de l'objet par une préposition. La deuxième structure est originale dans la mesure où elle contient un datif. Des différentes valeurs du datif français, la seule qui semble plausible ici est la valeur du datif de possession inaliénable. Ainsi la relation $Y / Z$ nous indique que l'expérienceur ne souhaite pas tant l'objet $Z$ mais le ressenti positif que $Z$ procure à $Y$, autrement dit, les effets de $Z$. Interprété de cette manière le premier énoncé exemple peut être expliqué de la manière suivante : Y peut se reposer quelques semaines et ça lui fera certainement du bien de se reposer, j'aimerais bien me reposer moi-aussi, ça me ferait du bien à moi aussi. Ce ne sont pas les jours de repos qui sont importants mais le bien qu'ils procurent.

Cette dynamique de l'envie peut être expliquée facilement à l'aide de la théorie

(9) 1430 pour le syntagme ressentir de la jalousie. Le verbe conjugué à la première personne : 5 résultats pour l'envie contre 182 pour la jalousie.

(10) 602 résultats pour éprouver de la jalousie. Le verbe conjugué à la première personne : 2 résultats pour 1'envie et 20 pour la jalousie.

(11) Le Robert Historique de la Langue Française (2000) précise qu'en ce qui concerne le verbe envier, déjà vers 1200 le sens affectif s'effaçait et la valeur du désir prenait le dessus.

(12) Le domaine conceptuel du penser semble par ailleurs activé dans pratiquement tous les cas où le domaine du vouloir est activé, à l'exception du concept de vouloir lui-même et des « volitions » non agentives comme justement celle exprimée par l'expression verbale avoir envie. Les implications entre penser et vouloir n'ont pas encore été suffisamment mises en évidence. 
des mondes telle qu'elle a été appliquée au domaine affectif par Zalizniak (1991): Dans le monde M1, actuel, je perçois la situation (Y possède Z), dans le monde projeté $\mathrm{M} 2$ je possède $\mathrm{Z}$ et je ressens quelque chose de bon. Dans le monde projeté on peut parler alors d'un affect positif, mais ce n'est plus de l'envie. Notons enfin que contrairement à la jalousie, il n'y a aucune contrainte sur l'objet Z, il n'est pas nécessaire que l'expérienceur soit en mesure de l'atteindre.

L'envie valorise l'objet Z. La jalousie le fait également d'une certaine façon mais cet aspect n'est pas profilé dans son cas. Dans l'envie, la valorisation est profilée et confirmée par l'existence de l'adjectif enviable, alors qu'il n'existe pas d'adjectif *jalousable. L'envie et ses lexèmes peuvent être également utilisés dans la publicité, par exemple «vos voisins vont vous envier... » alors que c'est très rare avec la jalousie. Notons également la possibilité de la coordination avec un affect positif, l'admiration, qui confirme la valorisation de l'objet :

J'admirais leur prestance, les enviais presque, loin d'imaginer les dangers qui les guettaient. (Frantext)

En un sens, je l'admirais et je l'enviais. (Frantext)

Nous venons de voir ce qui correspond à la composante cognitive de l'envie. Comme dans le cas de la jalousie, sont activés dans l'envie les domaines du penser et du vouloir. Par contre, il n'y a pas, comme cela a été déjà dit, de ressenti négatif et donc pas de ressenti tout court. L'envie n'a pas de composante physique non plus. On trouve des collocations du type rongé/dévoré par l'envie, mais les énoncés avec ces collocations sont interprétés le plus souvent comme l'expression de la valeur « désir non agentif».

En ce qui concerne l'axiologie externe de l'envie, elle est un peu floue. L'envie fait partie de sept péchés capitaux de la religion catholique, elle doit être foncièrement mauvaise, mais les locuteurs considèrent que l'envie n'est pas négative, contrairement à la jalousie. Ce jugement est confirmé par le fait que l'on peut produire des énoncés avec le verbe envier à la première personne, alors que c'est très rare avec la jalousie. C'est d'autant plus significatif que le verbe envier est un verbe transitif à la voie active qui présuppose, je le rappelle, un engagement de l'expérienceur bien plus fort que la structure essive être jaloux.

On observe par contre une différence en ce qui concerne le jugement axiologique externe des adjectifs jaloux et envieux. C'est envieux qui apparaît comme plus négatif, aussi bien lorsqu'il qualifie une personne en entier (un X envieux) que des comportements (cilenvieux, air envieux). Ce fait peut être probablement expliqué par la dynamique interne de la polysémie de la jalousie. L'adjectif jaloux, en emploi absolu, est interprété le plus souvent en terme de jalousie « conjugale ». Il semble que pour cette raison, l'adjectif envieux, par sa proximité sémantique se confond avec l'adjectif jaloux. Notons cependant que cela ne signifie pas une identité sémantique. Compte tenu des rapports des expressions de la jalousie, 1'adjectif jaloux, entrant dans la structure essive qui est l'expression expérientielle, est plus processuel, même lorsqu'il sert à qualifier, parce qu'il renvoie au procès affectif jalousie, alors que dans le cas de l'envie, l'adjectif ne fait pas partie de l'expression expérientielle, il est donc interprété simplement en terme de qualité.

Compte tenu des observations supra, la valeur de l'envie qui nous intéresse ici peut être explicitée de manière suivante.

Xpense :

quelque chose de bon (Z) est arrivé à cette personne (Y) 
je voudrais que des choses comme cela m'arrivent

si des choses comme cela m'arrivent je ressentirai quelque chose de bon

La jalousie et l'envie vont être comparées dans la section suivante, mais on peut d'emblée voir que le contenu sémantique de l'envie est beaucoup moins développé que celui de la jalousie.

\section{Les relations entre la jalousie et l'envie}

La jalousie et l'envie font partie des réactions à la situation dans laquelle une personne, autre que l'expérienceur, possède quelque chose (au sens matériel ou symbolique), que l'expérienceur aimerait avoir. Dans la mesure où elles ont le même schéma argumental $\mathrm{X}(\mathrm{Y} / \mathrm{Z})$, on peut considérer qu'il s'agit des variations autour de ce schéma (Koselak, 2008a). Leur proximité vient de là. Mais même s'il s'agit des réactions à une même situation, ces réactions sont différentes. Dans le cas de la jalousie et de l'envie, il ne s'agit même pas d'une conceptualisation différente d'une même situation, mais de deux conceptualisations de réactions différentes.

Reprenons tout d'abord les observations au sujet de deux concepts, sous la forme d'un tableau pour plus de clarté ( $c f$. page suivante).

La catégorisation de concepts mentaux est difficile parce qu'ils activent le plus souvent plusieurs domaines conceptuels. La jalousie et l'envie se recoupent partiellement dans la mesure où toutes les deux activent le domaine du penser et du vouloir, mais, comme nous l'avons vu, la jalousie active également les domaines de ressentir et de faire. Le champ lexical de la jalousie est moins riche que celui de l'envie. Observons la présence de deux adjectifs pour l'envie: envieux est centré sur l'expérienceur et enviable, sur l'objet. Un fait important, l'expression expérientielle de la jalousie est une structure essive et celle de l'envie est un verbe transitif à la voie active. Cela pose problème, comme nous le verrons, lors de la substitution, dans la mesure où les objets sont également introduits d'une manière différente et l'énoncé doit être partiellement restructuré. Enfin, l'axiologie externe de la jalousie est toujours négative alors celle de l'envie semble neutre.

Dans ma base de données extraite du Frantext, les noms jalousie et envie sont très rarement coordonnés, comme par exemple :

Je crois qu'un peu d'envie, et peut-être de jalousie se mêlait à notre étonnement. (Frantext).

Une telle coordination exclut, a priori, l'identité, mais n'exclut pas la proximité.

L'analyse et la comparaison sémantique de deux concepts montrent qu'ils sont différents, par contre, la substitution est possible dans la plupart de cas ${ }^{(13)}$. Tout d'abord les emplois nominaux :

Il était d'une jalousie qui empoisonnait nos relations.

Il était d'une envie qui empoisonnait nos relations.

Je les observais avec jalousie.

Je les observais avec envie.

Nous en sortir, coûte que coûte nous en sortir, coincés entre jalousie et admiration.

Nous en sortir, coûte que coûte nous en sortir, coincés entre envie et admiration.

(13) Tous les énoncés dans lesquels je procède aux substitutions proviennent de Frantext. 


\begin{tabular}{|c|c|c|}
\hline concept & la jalousie & l'envie \\
\hline $\begin{array}{l}\text { domaines } \\
\text { conceptuels } \\
\text { activés }\end{array}$ & penser, vouloir, ressentir, faire & penser, vouloir \\
\hline $\begin{array}{l}\text { expression } \\
\text { expérientielle }\end{array}$ & être jaloux : X est jaloux (de Z) de Y & $\begin{array}{c}\text { envier : } \mathrm{X} \text { envie } \mathrm{Z} \text { à } \mathrm{Y}_{\mathrm{DAT}} \\
\mathrm{X} \text { envie } \mathrm{Y}\end{array}$ \\
\hline $\begin{array}{l}\text { autres } \\
\text { expressions } \\
\text { linguistiques }\end{array}$ & $\begin{array}{l}\text { nom : jalousie } \\
\text { verbe : (jalouser) } \\
\text { adjectif : jaloux } \\
\text { adverbe : jalousement }\end{array}$ & $\begin{array}{l}\text { nom : envie } \\
\text { verbe : envier } \\
\text { adjectif : envieux, enviable } \\
\text { adverbe : envieusement }\end{array}$ \\
\hline $\begin{array}{l}\text { invariant } \\
\text { conceptuel } \\
\text { (explicitation) }\end{array}$ & $\begin{array}{l}X \text { a ressenti quelque chose parce que } X \text { a pensé quelque } \\
\text { chose } \\
\text { Parfois quelqu'un pense de quelqu'un d'autre: } \\
\text { «cette personne a quelque chose } \\
\text { je n'ai pas de chose de ce genre } \\
\text { je pense que c'est mauvais } \\
\text { je pense que je pourrais avoir quelque chose de ce } \\
\text { genre } \\
\text { je voudrais avoir quelque chose de ce genre » } \\
\text { quand quelqu'un pense cela, il ressent quelque chose } \\
\text { de mauvais } \\
\text { X a ressenti quelque chose comme cela } \\
\text { parce que X a pensé quelque chose comme cela }\end{array}$ & $\begin{array}{l}\text { X pense : } \\
\text { quelque chose de bon (Z) est } \\
\text { arrivé à cette personne (Y) } \\
\text { je voudrais que des choses } \\
\text { comme cela m'arrivent } \\
\text { si des choses comme cela } \\
\text { m'arrivent je ressentirai } \\
\text { quelque chose de bon }\end{array}$ \\
\hline $\begin{array}{l}\text { axiologie } \\
\text { externe }\end{array}$ & négative & neutre \\
\hline
\end{tabular}

Ces trois énoncés ont été jugés comme parfaitement plausibles ${ }^{(14)}$ par mes informateurs, seulement dans la première paire l'énoncé avec jalousie est jugé meilleur que celui avec envie. Les deux autres énoncés ont été considérés comme égaux. Notons que ces énoncés contiennent très peu de spécifications sémantiques de concepts étudiés et on peut très bien y introduire d'autres concepts sans que les énoncés cessent d'être acceptables. Bien évidemment le sens des énoncés change nécessairement, les différences sont celles que l'on retrouve dans le tableau récapulatif. Par contre, la substitution s'avère problématique lorsque l'environnement sémantique est spécifique, comme par exemple dans :

Une fois goûtée, dégustée, savourée la jalousie verte des collègues, une panique m'envahit.

Une fois goûtée, dégustée, savourée l'envie verte des collègues, une panique m'envahit.

(14) Pour de raisons méthodologiques je ne marque pas les énoncés comme étant incorrects ou problématiques, l'acceptabilité est toujours une question de degré. Il est par contre plus intéressant de réfléchir sur la plausibilité d'occurrences des énoncés dans la mesure où les énoncés tout à fait corrects peuvent être jugés comme étant non plausibles, donc pas susceptible d'être produits dans les conditions normales. Le terme «conditions normales » est flou, comme l'est la notion même de normalité. Je comprends par «conditions normales" la situation dans laquelle l'énoncé interprété ne nécessite pas de justifications contextuelles complexes. 
L'énoncé avec envie est bizarre, probablement parce qu'il active la métaphore spécifique de la jalousie "jalousie est parfois comme une maladie », la couleur verte étant associée en français à la maladie et cette métaphore est incompatible avec l'envie. Notons à ce titre que les expressions (être) rongéldévoré par envie sont interprétées le plus souvent comme exprimant la valeur "désir non-agentif » de l'envie. La première observation qui se dégage est que la substitution est possible sans réserve dans les énoncés peu spécifiques. Du moment où l'on tente des substitutions dans des environnements plus spécifiques, des problèmes apparaissent.

Passons maintenant à la substitution des structures expérientielles. Comme cela a déjà été dit, cette substitution nécessite une réorganisation partielle de l'énoncé. La motivation du sens par la forme fait que ces énoncés se distinguent d'emblée par un degré d'engagement de l'expérienceur.

J'enviais cette patiente qui, sans difficulté, pouvait situer chronologiquement avec leurs dates les moindres événements de sa vie.

J'étais jaloux de cette patiente qui, sans difficulté, pouvait situer chronologiquement avec leurs dates les moindres événements de sa vie.

Parfois elle enviait à certains de ses amis leurs parents mais, aussitôt, se le reprochait.

Parfois elle était jalouse des parents de certains de ses amis mais, aussitôt, se le reprochait.

Nous possédons des équipes de chercheurs que le monde entier nous envie, qu'il cherche souvent, à prix d'or, à nous arracher.

Nous possédons des équipes de chercheurs dont tout le monde est jaloux....

Dans ces trois énoncés, la substitution ne pose aucun problème, seulement dans le dernier, les informateurs considèrent que le terme jaloux est peut-être « trop fort ». Il s'agit ici aussi des environnements sémantiques peu spécifiques dans lesquels la jalousie et l'envie unissent des actants. Mais même ce lien établi est différent dans la mesure où son expression linguistique est différente. Le sens des énoncés est changé aussi bien par l'orientation axiologique, l'expérienceur de la jalousie est jugé comme " mauvais », que par l'engagement de l'expérienceur dans le procès (plus grand dans l'envie).

Les substitutions des adjectifs jaloux et envieux sont possibles dans pratiquement tous les énoncés, ce qui n'est pas étonnant compte tenu de ce qui a été dit au sujet de ces deux adjectifs.

\section{Bilan}

La jalousie et l' envie sont-elles finalement synonymes ou non ? La démarche appliquée ici a été de décrire le contenu conceptuel et de le comparer. Nous avons vu que les domaines conceptuels activés par la jalousie et l'envie ne se recouvraient que partiellement. La jalousie est un affect et l'envie ne l'est pas, lajalousie est plus complexe et elle active plus de domaines conceptuels que l'envie, mais les domaines activés par l'envie (penser et vouloir) le sont également par la jalousie.

Nous avons vu en outre que la jalousie et l'envie étaient des variations autour d'un même schéma, il y a également une partie de leur contenu qui est très proche. Il existe très peu de moyens formalisés pour décider si un couple de concepts est synonyme. Observons par exemple les implications des deux concepts ( $c f$. Cruse, 2002) : 
Il lui enviait sa gloire mais il n'en était pas jaloux.

Le premier énoncé semble pour le moins bizarre si l'explication de l'envie donnée supra est juste, la jalousie impliquant, du moins partiellement, l'envie. Par contre, le deuxième énoncé ne pose aucun problème, la négation de la jalousie signifie la négation du ressenti négatif. Il y a donc une dissymétrie. Cette dissymétrie est certainement due au fait que la jalousie est un concept plus complexe et plus défini que l'envie. La jalousie, par sa complexité, peut impliquer l'envie parce qu'une telle implication ne nie pas les caractéristiques de l'envie, d' où la bizarrerie du premier énoncé.

La proximité de ces deux concepts peut être schématisée de la manière suivante:

\begin{tabular}{|l|l|l|}
\hline \multicolumn{1}{|l|}{ ENVIE } & $\begin{array}{l}\text { cette personne a quelque chose } \\
\text { je n'ai pas de chose de ce genre / } \\
\text { quelque chose de bon est arrivé à } \\
\text { cette personne }\end{array}$ & $\begin{array}{l}\text { je pense que c'est mauvais } \\
\text { je pense que je pourrais } \\
\text { avoir quelque chose de ce } \\
\text { genre }\end{array}$ \\
$\begin{array}{l}\text { Si des choses } \\
\text { comme cela m'ar- } \\
\text { rivent je ressenti- } \\
\text { rai quelque chose } \\
\text { de bon }\end{array}$ & $\begin{array}{l}\text { je voudrais avoir quelque chose } \\
\text { de ce genre / je voudrais que des } \\
\text { choses comme cela m'arrivent }\end{array}$ & $\begin{array}{l}\text { je ressens quelque chose de } \\
\text { mauvais }\end{array}$ \\
\hline
\end{tabular}

Les formulations de la partie centrale sont légèrement différentes pour la jalousie et pour l'envie dans la mesure où lajalousie profile la relation à un objet, alors que l'envie plutôt les effets de la possession d'un objet, on peut cependant partir du principe que ces différences sont minimales. Le seul trait spécifique à l'envie: « Si des choses comme cela m'arrivent je ressentirai quelque chose de bon » est, logiquement, sous-entendu par la jalousie, même s'il ne fait pas partie du contenu sémantique. L'envie est donc moins définie sémantiquement que la jalousie. On ne peut pas dire pour autant que l'envie soit un genre d'hypéronyme pour la jalousie, on ne peut définir ni l'envie à l'aide de jalousie ni le contraire : la jalousie n'est pas un genre d'envie et l'envie n'est pas un genre de jalousie. Il est difficile également de considérer que la jalousie et l'envie soient des co-hyponymes dans la mesure où, comme cela a déjà été dit, il n'est pas possible de trouver un hypéronyme commun. La jalousie et l'envie sont unies par la proximité thématique : il s'agit des variations de réponse de la même situation : $\mathrm{X}(\mathrm{Y} / \mathrm{Z})$, cependant il s'agit de concepts de réaction complètement différents. La substitution change nécessairement le sens et ceci à au moins deux niveaux : sémantique à proprement dit et le niveau de l'engagement de l'expérienceur dans le processus mental en question. Cruse (2002) cité supra posait qu'il existe un consensus intuitif pour décider si tel ou tel couple d'unités linguistiques était synonymes. Or, il semble qu'en ce qui concerne la jalousie et l'envie, il n'y a pas un tel consensus. Il faudrait encore confirmer ce fait par une enquête systématique mais les locuteurs interrogés sont très partagés. 
En conclusion, je dirai donc qu'une proximité sémantique n'implique pas nécessairement une relation de sens répertoriée et décrite comme l'est la synonymie. Les relations dans le lexique sont complexes et les relations de sens procèdent nécessairement à des schématisations de cette complexité. Les relations apparaissant comme évidentes lorsque l'on les observe dans le lexique concernant les objets du monde « matériel» le sont moins dans les domaines conceptuels mental et affectif. La difficulté de réduire les rapports entre la jalousie et l'envie à une relation de sens comme la synonymie n'est pas une exception, j'ai montré ailleurs (Koselak, 2007b) que l'on retrouve également la difficulté lorsque l'on postule l'antonymie des termes : les relations entre l'amour et la haine sont plus complexes que des relations d'antonymie.

\section{Indications bibliographiques}

BARTMIŃSKI, J. (2003) : « Miejsce wartości w językowym obrazie świata », in BARTMIŃSKI, J. (ed), Język w kręgu wartości, Lublin, Wydawnictwo UMCS, pp. 7-14.

CRUSE, D. A. (2002) : « Paradigmatic relations of inclusion and identity III : Synonymy », in D.A. Cruse, F. Hundsnurscher, M. Job, Lexicologie. Lexicology, Berlin/New York, Walter de Gruyter.

HARKINS, J., WIERZBICKA, A. (eds) (2001) : Emotions in Crosslinguistic Perspective, Berlin/New York, Mouton de Gruyter.

KOSELAK, A. (2003) : «La sémantique naturelle d'Anna Wierzbicka et les enjeux interculturels », Communications, 4, pp. 83-97.

- $(2007 \mathrm{a})$ : « Sources et tradition polonaise en linguistique cognitive », CORELA.

— (2007b) : Sémantique de quelques " mauvais » sentiments en français et en polonais, Thèse de doctorat de l'Université Paul Verlaine de Metz, sous dir. de C. Masseron et A. Petitjean.

- (2008a) : «Un modèle descriptif des affect. L'exemple de la jalousie et de zazdrość », Konferencja Naukowa : Metody analizy leksykalnej : założenia teoretyczne i zastosowania praktyczne, Białystok, 9-11.10.2008, communication orale, (Actes à paraître).

- (2008b) : «Cette personne a quelque chose que je n'ai pas. Une approche contrastive de réactions du type de jalousie », in Durand, J. Habert, B., Laks, B. (éds.) Congrès Mondial de Linguistique Française - CMLF'08, 2008, Institut de Linguistique Française, pp. 2085-2100 http ://www.linguistiquefrancaise.org/index.php?option=article\&access $=$ standard\&Itemid=129\&url=/articles/cmlf/abs/2008/01/ cmlf08050/cmlf08050.html.

— (à paraître) : Review of Semantic Primes and Universal Grammar : « Empirical evidence from the Romance languages », Ed. by BERT PEETERS. (Studies in language companion series 81) Amsterdam/Philadelphia: John Benjamins, WORD.

LE PETIT ROBERT. Dictionnaire de la langue française, 1993 
MACLEAN, P.D. (1980) : « The triune brain », in Rorty O.A. (ed), Explaining emotions, Berkeley/Los Angeles/London, University of California Press, pp. 9-36.

MACKIEWICZ, J. (1999) : « Co to jest językowy obraz świata ?», Etnolingwistyka, 11, pp. 7-24.

NIKLAS-SAlminen, A. (1997) : La lexicologie, Paris, Armand Colin.

PEETERS, B. (2002) : «La "métalangue sémantique naturelle" au service de l'étude du transculturel », Travaux de linguistique 45, Gand, pp. 83-101.

— (2006) (ed) : «Semantic Primes and Universal Grammar : Empirical evidence from the Romance languages », (Studies in language companion series 81) Amsterdam/Philadelphia : John Benjamins.

WIERZBICKA, A. (1985) : Lexicography and conceptual analysis, Karoma Publishers, Tucson.

— (1988) : The Semantics of Grammar, Amsterdam/Philadelphia, John Benjamins.

- (1999b) : Emotions across Languages and Cultures. Diversity and universals, Paris/Cambridge : Cambridge University Press/Editions de la Maison des Sciences de l'Homme.

ZALIZNIAK, A. (1991) : «De la nature du regret », BULAG, 17, 67-75.

\section{PRATIQUES}

\section{NUMÉRO PRÉCÉDENT}

$n^{\circ} 139 / 140$ (décembre 2008)

\section{Linguistique populaire ?}

\section{Coordination: Marie-Anne Paveau et Guy Achard-Bayle}

Il s'agit dans ce numéro de rendre compte d'abord de l'absence en France d'un champ identifié comme « la linguistique populaire " par rapport aux domaines anglo-saxon et allemand qui possèdent des disciplines institutionnellement reconnues et explicitement désignées (folk linguistics et Volklinguistik). Des chercheurs étrangers (américains et européens) sollicités pour le numéro permettront de mettre en perspective les différents enjeux de cette question.

Après avoir montré que les traditions nationales et l'histoire des sciences du langage dans les différents pays sont à l'origine des approches très différentes des analyses spontanées des «nonlinguists» selon l'expression de Preston et Niedzielski, on se propose d'explorer voire de définir ce qui relèverait du champ de la linguistique populaire dans le domaine francophone par rapport à des domaines connexes et des problématiques affines (les travaux sur la norme, sur le métalangage, le champ non savant du « purisme » à la française, etc.).

Il s'agira également, dans une perspective didactique, de questionner les relations et les apports de la linguistique populaire à la linguistique générale appliquée pour la connaissance et l'enseignement-apprentissage de la langue : on sait en effet à quel point les représentations dites «spontanées » ou « culturelles » de la langue sont importantes, à la fois chez les apprenants et les enseignants, dans l'enseignement-apprentissage du français et l'on s'interrogera donc sur la place, la validité et l'efficacité pédagogique des pratiques relevant de la linguistique populaire. 